\title{
Strong convergence of a modified viscosity iteration for common zeros of a finite family of accretive mappings
}

\author{
Yuanheng Wang ${ }^{\mathrm{a}, *}$, Yan Li ${ }^{\mathrm{b}}$, Chanjuan Pan ${ }^{\mathrm{a}}$ \\ ${ }^{a}$ Department of Mathematics, Zhejiang Normal University, 321004 Zhejiang, China. \\ ${ }^{b}$ Department of Basic Science, Nanyang Polytechnic Institute, 473000 Henan, China.
}

Communicated by Y. H. Yao

\begin{abstract}
A new modified iterative scheme $\left\{x_{n}\right\}$ is given for the viscosity approximating a common zero of a finite family of accretive mappings $\left\{A_{i}\right\}$ in reflexive Banach spaces with a weakly continuous duality mapping $J$ in the present paper. Under certain conditions, we prove the strong convergence of the sequence $\left\{x_{n}\right\}$. The results here extend and improve the corresponding recent results of some other authors. (c)2017 All rights reserved.
\end{abstract}

Keywords: Viscosity approximation method, accretive mapping, common zero, strong convergence, reflexive Banach space. 2010 MSC: 47H10, 47H09, 47J25.

\section{Introduction}

Let $E$ be a real Banach spaces and $J$ be the normalized duality mapping defined by

$$
J(x)=\left\{f \in E^{*}:\langle x, f\rangle=\|x\|\|f\|,\|x\|=\|f\|\right\}, \quad \forall x \in E,
$$

where $\langle\cdot, \cdot\rangle$ denotes the duality pairing and $E^{*}$ denotes the dual space of $E$. It is well-known that if $E^{*}$ is strictly convex then $\mathrm{J}$ is single-valued.

Assume that $\mathrm{K}$ is a nonempty closed convex subset of $\mathrm{E}$. A mapping $\mathrm{T}: \mathrm{K} \rightarrow \mathrm{K}$ is contractive on $\mathrm{K}$ if there exists a constant $\alpha \in(0,1)$ such that

$$
\|f(x)-f(y)\| \leqslant \alpha\|x-y\|, \quad \forall x, y \in K .
$$

$\Pi_{K}$ denotes the collection of all contractive mappings on $K$. Let $\Pi_{K}=\{f: f$ is a contractive mapping on $K\}$. Let $T$ be a operator (possibly multivalued) with domain with domain $D(T)$ and range $R(T)$ in $E$. $T$ is said to be nonexpansive if

$$
\|T(x)-T(y)\| \leqslant\|x-y\|, \quad \forall x, y \in K
$$

\footnotetext{
${ }^{*}$ Corresponding author

Email addresses: yhwang@zjnu.cn (Yuanheng Wang), lirongfu703@sina.com (Yan Li), cjpanzjnu@163.com (Chanjuan Pan) doi:10.22436/jnsa.010.09.18
} 
We use $F(T)$ to denote the set of fixed points of $T$, that is $F(T)=\{x: x \in K, x=T x\}$.

If there exists a $j\left(x_{1}-x_{2}\right) \in J\left(x_{1}-x_{2}\right)$ such that $\left\langle y_{1}-y_{2}, j\left(x_{1}-x_{2}\right)\right\rangle \geqslant 0$ for each $x_{i} \in D(T)$, then $T$ is said to be accretive. $T$ is said to satisfy the range condition if for all $r>0$, such that $\operatorname{cl}(D(T)) \subset R(I+r T)$. $T$ is said to be $m$-accretive if $T$ is an accretive operator and $R(I+r T)=E$ for all $r>0$. If an accretive operator $T$ satisfies the range condition, then for all $r>0$, we define the mapping $J_{r}^{T}: R(I+r T) \rightarrow D(T)$ by $J_{r}^{\top}=(I+r T)^{-1}, J_{r}^{\top}$ is called the resolvent operator of $T$. We know that $J_{r}^{\top}$ is nonexpansive and $F\left(J_{r}^{\top}\right)=N(T)$ for all $r>0$, where $N(T)=T^{-1}(0)=\{x \in D(T): 0 \in T x\}, F\left(J_{r}^{\top}\right)=\left\{x \in E: J_{r}^{\top} x=x\right\}$.

Kim and $\mathrm{Xu}[8]$ introduced an iterative sequence given by

$$
x_{n+1}=\alpha_{n} u+\left(1-\alpha_{n}\right) J_{r_{n}} x_{n},
$$

under certain conditions, they showed the iterative sequence $\left\{x_{n}\right\}$ converges strongly to a zero of $A$ in the uniformly smooth Banach spaces. Xu [15] extended Kim and Xu's result [8] from a uniformly smooth Banach space to a reflexive Banach space which has a weakly continuous duality mapping.

Zegeye and Shahzad [18] extended Xu's result $[8,15]$ from an m-accretive operator to a finite family of $m$-accretive operators. Let the sequence $\left\{x_{n}\right\}$ defined by

$$
x_{n+1}=\alpha_{n} u+\left(1-\alpha_{n}\right) S_{r} x_{n}, \quad n \geqslant 0,
$$

where $S_{r}=a_{0} I+a_{1} J_{A_{1}}+a_{2} J_{A_{2}}+\cdots+a_{r} J_{A_{r}}$ with $J_{A_{r}}=\left(I+A_{i}\right)^{-1}$ for $0<a_{i}<1, i=0,1, \cdots, r$, $\sum_{i=0}^{r} a_{i}=1$. Under certain conditions, they proved $\left\{x_{n}\right\}$ converges strongly to a common solution of the equations $A_{i} x=0$, for $i=1,2, \cdots, r$.

Moudafi [10] first proposed viscosity approximation method in 2000, since then many authors investigated the viscosity iterative sequence, see $([2-4,7,14,16,17])$, Chen and Zhu $[3,4]$ used contractive mapping and the resolvent $J_{r_{n}}$ of $m$-accretive operator $A$ to construct viscosity iterative sequence $\left\{x_{n}\right\}$

$$
x_{n+1}=\alpha_{n} f\left(x_{n}\right)+\left(1-\alpha_{n}\right) J_{r_{n}} x_{n} .
$$

Under certain conditions, they proved strongly convergence of the iterative sequence $\left\{x_{n}\right\}$ in the framework of a uniformly smooth Banach space and a reflexive Banach space which has a weakly continuous duality mapping, respectively.

Very recently Wang et al. [11] introduced a brand new iterative scheme $\left\{x_{n}\right\}$ by composite approximation method for finding a common zero of two accretive operators $A$ and B in Banach spaces.

$$
\left\{\begin{aligned}
y_{n} & =\beta_{n} J_{r_{n}}^{B} x_{n}+\left(1-\beta_{n}\right) J_{r_{n}}^{A} x_{n}, \\
x_{n+1} & =\alpha_{n} x_{n}+\left(1-\alpha_{n}\right) y_{n}, \quad n \geqslant 0
\end{aligned}\right.
$$

which converges weakly to a common zero of two accretive operators A and B under certain conditions.

Motivated by the above results, we study the following iterative sequence:

$$
\left\{\begin{aligned}
y_{n} & =a_{1} J_{r_{n}}^{A_{1}} x_{n}+a_{2} J_{r_{n}}^{A_{2}} x_{n}+\cdots+a_{l} J_{r_{n}}^{A_{l}} x_{n}, \\
x_{n+1} & =\alpha_{n} f\left(x_{n}\right)+\left(1-\alpha_{n}\right) y_{n}, \quad n \geqslant 0 .
\end{aligned}\right.
$$

We prove the iterative sequence $\left\{x_{n}\right\}$ defined by (1.1) converges strongly to a common zero of a finite family of accretive operators in a reflexive Banach space which has a weakly continuous duality mapping. The results in this paper improve and extend some recent corresponding results of other authors.

\section{Preliminaries}

The following definitions and lemma are needed in order to prove our results.

A Banach space $E$ is called strictly convex if there exist $a_{i} \in(0,1), i=1,2, \cdots, l$, such that $\sum_{i=1}^{l} a_{i}=1$. when $\left\|x_{1}\right\|=\left\|x_{2}\right\|=1$, we have $\left\|a_{1} x_{1}+a_{2} x_{2}\right\|<1$ for $x_{i} \in E$. In a strictly convex Banach space $E$, if for 
$x_{i} \in E, a_{i} \in(0,1), i=1,2, \cdots, l$ and $\left\|x_{1}\right\|=\left\|x_{2}\right\|=\cdots=\left\|x_{l}\right\|=\left\|a_{1} x_{1}+a_{2} x_{2}+\cdots+a_{l} x_{l}\right\|$, such that $\sum_{i=1}^{l} a_{i}=1$, then we have that $x_{1}=x_{2}=\cdots=x_{l}$.

A continuous strictly increasing function $\varphi:[0, \infty) \rightarrow[0, \infty)$ is said to be a gauge if $\varphi(0)=0$ and $\varphi(\mathrm{t}) \rightarrow \infty$ as $\mathrm{t} \rightarrow \infty$. The $\mathrm{J}_{\varphi}: \mathrm{E} \rightarrow \mathrm{E}^{*}$ is defined by

$$
\mathrm{J}_{\varphi}(x)=\left\{x^{*} \in \mathrm{E}^{*}:\left\langle x, x^{*}\right\rangle=\|x\| \varphi(\|x\|),\left\|x^{*}\right\|=\varphi(\|x\|)\right\}, \quad \forall x \in \mathrm{E},
$$

is called a duality mapping associated to a gauge $\varphi$.

A Banach space $E$ is said to be a weakly continuous duality mapping if there exists a gauge $\varphi$ for which the duality mapping $\mathrm{J}_{\varphi}$ is single-valued and weak-to-weak ${ }^{*}$ sequentially continuous. Such as, $l^{p}$ has a weakly continuous duality mapping for all $1<p<\infty$, with gauge $\varphi(t)=t^{p-1}$. Let

$$
\phi(t)=\int_{0}^{t} \varphi(\tau) d \tau, \quad t \geqslant 0
$$

and

$$
\mathrm{J}_{\varphi}(x)=\partial \phi(\|x\|), \quad \forall x \in \mathrm{E} .
$$

Then [9] and $\partial$ denote the subdifferential in the sense of convex analysis.

Lemma 2.1 ([19]). Let a Banach space E have a weakly continuous duality mapping $\mathrm{J}_{\varphi}$ with a gauge $\varphi$.

(i) The following inequality holds

$$
\phi(\|x+y\|) \leqslant \phi(\|x\|)+\left\langle y, J_{\varphi}(x+y)\right\rangle, \quad \forall x, y \in E .
$$

(ii) If $\left\{x_{n}\right\} \subset E, x \rightarrow E$, then the following identity holds

$$
\limsup _{n \rightarrow \infty} \phi\left(\left\|x_{n}-y\right\|\right)=\limsup _{n \rightarrow \infty} \phi\left(\left\|x_{n}-x\right\|\right)+\phi(\|y-x\|), \quad \forall x, y \in E,
$$

where $\rightarrow$ and $\rightarrow$ denote the weak convergence and the strong convergence, respectively.

Lemma 2.2 ([13]). Let $\left\{\alpha_{n}\right\}$ be a sequence of nonnegative real numbers satisfying the condition

$$
\alpha_{\mathrm{n}+1} \leqslant\left(1-\gamma_{\mathrm{n}}\right) \alpha_{\mathrm{n}}+\gamma_{\mathrm{n}} \sigma_{\mathrm{n}}, \quad \forall \mathrm{n} \geqslant 0,
$$

where $\left\{\gamma_{\mathrm{n}}\right\} \subset(0,1)$ and $\left\{\sigma_{\mathrm{n}}\right\}$ such that

(i) $\lim _{n \rightarrow \infty} \gamma_{n}=0$ and $\sum_{n=0}^{\infty} \gamma_{n}=\infty$;

(ii) either $\limsup _{n \rightarrow \infty} \sigma_{n} \leqslant 0$ or $\sum_{n=0}^{\infty}\left|\gamma_{n} \sigma_{n}\right|<\infty$.

Then $\left\{\alpha_{n}\right\}$ converges to zero.

Lemma 2.3 (The resolvent identity [1]). For $\lambda, \mu>0$, and each $x \in E$, there holds the identity:

$$
\mathrm{J}_{\lambda} x=\mathrm{J}_{\mu}\left(\frac{\mu}{\lambda} x+\left(1-\frac{\mu}{\lambda}\right) J_{\lambda} x\right) .
$$

Lemma 2.4 ([12]). Let $\mathrm{E}$ be a reflexive Banach space, $\mathrm{C}$ be a nonempty closed convex subset of $\mathrm{E}$, and $\mathrm{T}: \mathrm{C} \rightarrow \mathrm{E}$ an asymptotically contractive with Lipschitz continuous mapping. Suppose that $\mathrm{E}$ admits a weakly sequentially continuous duality mapping. Then the mapping $\mathrm{I}-\mathrm{T}$ is demiclosed on $\mathrm{C}$, where $\mathrm{I}$ is the identity mapping, i.e., if $x_{n} \rightarrow x$ and $\left\|x_{n}-T x_{n}\right\| \rightarrow 0$, then $x=T x$.

Lemma 2.5 ([5]). Let $\mathrm{E}$ be a reflexive Banach space which has a weakly continuous duality mapping $\mathrm{J}_{\varphi}$ with gauge $\varphi$. Suppose $\mathrm{C}$ is a nonempty closed convex subset of $\mathrm{E}$, and $\mathrm{T}: \mathrm{C} \rightarrow \mathrm{C}$ is a nonexpansive mapping. Let $\mathrm{f}: \mathrm{C} \rightarrow \mathrm{C}$ be a contractive mapping, for $\mathrm{t} \in(0,1), x_{\mathrm{t}}=\mathrm{tf}\left(\mathrm{x}_{\mathrm{t}}\right)+(1-\mathrm{t}) \mathrm{T} \mathrm{x}_{\mathrm{t}}$. Then $\mathrm{T}$ has a fixed point if only if $\left\{\mathrm{x}_{\mathrm{t}}\right\}$ remains 
bounded as $\mathrm{t} \rightarrow 0^{+}$and in this case, $\left\{x_{\mathrm{t}}\right\}$ converges strongly to a fixed point of $\mathrm{T}$ as $\mathrm{t} \rightarrow 0^{+}$. If we define a mapping $\mathrm{Q}: \Pi_{\mathrm{c}} \rightarrow \mathrm{F}(\mathrm{T})$ by $\mathrm{Q}(\mathrm{f})=\lim _{\mathrm{t} \rightarrow 0} x_{\mathrm{t}}, \mathrm{f} \in \Pi_{\mathrm{c}}$, then $\mathrm{Q}(\mathrm{f})$ solves the variational inequality

$$
\left\langle(I-f) Q(f), J_{\varphi}(Q(f)-p)\right\rangle \leqslant 0, \quad \forall p \in F(T) .
$$

Lemma 2.6. Let $\mathrm{C}$ be a nonempty closed convex subset of a strictly convex Banach space $\mathrm{E} . \mathrm{Let}_{i}: \mathrm{C} \rightarrow$ $E, i=1,2, \cdots, l$ be a finite family of accretive operators such that $\bigcap_{i=1}^{l} N\left(A_{i}\right) \neq \emptyset$ satisfying the range conditions $\operatorname{cl}\left(D\left(A_{i}\right)\right) \subset C \subset \bigcap_{r_{n}>0} R\left(I+r_{n} A_{i}\right), i=1,2, \cdots, l$. Let $a_{1}, a_{2}, \cdots$, $a_{l}$ be real numbers in $(0,1)$ such that $\sum_{i=1}^{l} a_{i}=1$ and $S_{r_{n}}=a_{1} J_{r_{n}}^{A_{1}}+a_{2} J_{r_{n}}^{A_{2}}+\cdots+a_{l} J_{r_{n}}^{A_{l}}$, where $J_{r_{n}}^{A_{i}}=\left(I+r_{n} A_{i}\right)^{-1}$ and $r_{n}>0$. Then $S_{r_{n}}$ is nonexpansive and $\mathrm{F}\left(\mathrm{S}_{\mathrm{r}_{\mathrm{n}}}\right)=\bigcap_{\mathrm{i}=1}^{\mathrm{l}} \mathrm{N}\left(\mathrm{A}_{\mathrm{i}}\right)$.

Proof. Since each $A_{i}$ satisfies the range conditions for any $i \in\{1,2, \cdots, l\}$, we have that $J_{r_{n}}^{A_{i}}$ is well-defined nonexpansive mappings from $R\left(I+r_{n} A_{i}\right)$ to $C$ with $F\left(J_{r_{n}}^{A_{i}}\right)=N\left(A_{i}\right)$. For any $x, y \in \bigcap_{i=1}^{l} R\left(I+r_{n} A_{i}\right)$, and for all $r_{n}>0$, we have

$$
\begin{aligned}
\left\|S_{r_{n}} x-S_{r_{n}} y\right\| & =\left\|a_{1} J_{r_{n}}^{A_{1}} x+a_{2} J_{r_{n}}^{A_{2}} x+\cdots+a_{l} J_{r_{n}}^{A_{l}} x-a_{1} J_{r_{n}}^{A_{1}} y-a_{2} J_{r_{n}}^{A_{2}}-\cdots-a_{l} J_{r_{n}}^{A_{l}}\right\| \\
& \leqslant \sum_{i=1}^{l} a_{i}\left\|J_{r_{n}}^{A_{i}} x-J_{r_{n}}^{A_{i}} y\right\| \leqslant \sum_{i=1}^{l} a_{i}\|x-y\|=\|x-y\| .
\end{aligned}
$$

So we have know that $S_{r_{n}}$ is nonexpansive. Since $F\left(J_{r_{n}}^{A_{i}}\right)=N\left(A_{i}\right)$, so

$$
\bigcap_{i=1}^{l} N\left(A_{i}\right)=\bigcap_{i=1}^{l} F\left(J_{r_{n}}^{A_{i}}\right) \subseteq F\left(S_{r_{n}}\right) .
$$

Next we will show that $F\left(S_{r_{n}}\right) \subseteq \bigcap_{i=1}^{l} F\left(J_{r_{n}}^{A_{i}}\right)$. Let $p \in \bigcap_{i=1}^{l} F\left(J_{r_{n}}^{A_{i}}\right), q \in F\left(S_{r_{n}}\right)$, then

$$
\begin{aligned}
\|q-p\| & =\left\|a_{1}\left(J_{r_{n}}^{A_{1}} q-p\right)+a_{2}\left(J_{r_{n}}^{A_{2}} q-p\right)+\cdots+a_{l}\left(J_{r_{n}}^{A_{l}} q-p\right)\right\| \\
& \leqslant \sum_{i=1}^{l} a_{i}\left\|J_{r_{n}}^{A_{i}} q-p\right\| \leqslant \sum_{i=1}^{l} a_{i}\|q-p\|=\|q-p\| .
\end{aligned}
$$

From (2.1) we obtain

$$
\begin{aligned}
\|q-p\| & =\sum_{i=1}^{l-1} a_{i}\|q-p\|+a_{l}\left\|J_{r_{n}}^{A_{l}} q-p\right\| \\
& =\left(1-a_{l}\right)\|q-p\|+a_{l}\left\|J_{r_{n}}^{A_{l}} q-p\right\|,
\end{aligned}
$$

and so

$$
\|q-p\|=\left\|J_{r_{n}}^{A_{l}} q-p\right\|
$$

Similarly we obtain that

$$
\|q-p\|=\left\|J_{r_{n}}^{A_{1}} q-p\right\|=\left\|J_{r_{n}}^{A_{2}} q-p\right\|=\cdots=\left\|J_{r_{n}}^{A_{l}} q-p\right\| .
$$

From (2.1) we get that

$$
\|q-p\|=\left\|a_{1}\left(J_{r_{n}}^{A_{1}} q-p\right)+a_{2}\left(J_{r_{n}}^{A_{2}} q-p\right)+\cdots+a_{l}\left(J_{r_{n}}^{A_{l}} q-p\right)\right\|,
$$

and by the strictly convexity of $E$, we have that

$$
q-p=J_{r_{n}}^{A_{1}} q-p=J_{r_{n}}^{A_{2}} q-p=\cdots=J_{r_{n}}^{A_{l}} q-p .
$$


So $J_{r_{n}}^{A_{i}} q=q$, for $i=1,2, \cdots, l$, which implies $q \in \bigcap_{i=1}^{l} F\left(J_{r_{n}}^{A_{i}}\right)$. Therefore

$$
F\left(S_{r_{n}}\right) \subseteq \bigcap_{i=1}^{l} F\left(J_{r_{n}}^{A_{i}}\right),
$$

then we have

$$
F\left(S_{r_{n}}\right)=\bigcap_{i=1}^{l} F\left(J_{r_{n}}^{A_{i}}\right)=\bigcap_{i=1}^{l} N\left(A_{i}\right) .
$$

\section{Main results}

Theorem 3.1. Assume that $\mathrm{E}$ is strictly convex reflexive Banach space with a weakly continuous duality mapping $\mathrm{J}_{\varphi}$ associated to a gauge $\varphi$. Let $\mathrm{K} \subset \mathrm{E}$ be a nonempty closed convex subset and $\mathrm{f}: \mathrm{K} \rightarrow \mathrm{K}$ be a contractive mapping with the contractive coefficient $\alpha \in(0,1)$. Let $A_{i}: K \rightarrow E, i=1,2, \cdots, l$ be a finite family of accretive operators such that $\bigcap_{i=1}^{l} \mathrm{~N}\left(A_{i}\right) \neq \emptyset$ satisfying the range conditions $\operatorname{cl}\left(\mathrm{D}\left(A_{i}\right)\right) \subset \mathrm{K} \subset \bigcap_{\mathrm{r}_{n}>0} \mathrm{R}\left(\mathrm{I}+\mathrm{r}_{\mathrm{n}} A_{i}\right), i=1,2, \cdots, l$, $J_{r_{n}}^{A_{i}}=\left(I+r_{n} A_{i}\right)^{-1}$ for $i=1,2, \cdots$, l. For any $x_{0} \in K$, let $\left\{x_{n}\right\}$ be defined by the formula (1.1). Assume that $0<a_{i}<1$, for $i=1,2, \cdots, l, \sum_{i=1}^{l} a_{i}=1$ and $\left\{\alpha_{n}\right\} \subset(0,1),\left\{\gamma_{n}\right\} \subset(0, \infty)$ which satisfy the following conditions:

(i) $\lim _{n \rightarrow \infty} \alpha_{n}=0, \sum_{n=0}^{\infty} \alpha_{n}=\infty, \sum_{n=0}^{\infty}\left|\alpha_{n+1}-\alpha_{n}\right|<\infty$;

(ii) $\gamma_{n} \geqslant \epsilon$ for $n \geqslant 0, \sum_{n=0}^{\infty}\left|\gamma_{n+1}-\gamma_{n}\right|<\infty$. Then $\left\{x_{n}\right\}$ converges strongly to a common zero of $A_{i}, i=$ $1,2, \cdots, l$.

Proof.

Step 1. We show that $\left\{x_{n}\right\}$ is bounded. Indeed, we can take a point $p \in \bigcap_{i=1}^{l} N\left(A_{i}\right)=\bigcap_{i=1}^{l} F\left(J_{r_{n}}^{A_{i}}\right)$. So from (1.1), we have

$$
\begin{aligned}
\left\|y_{n}-p\right\| & =\left\|a_{1} J_{r_{n}}^{A_{1}} x_{n}+a_{2} J_{r_{n}}^{A_{2}} x_{n}+\cdots+a_{l} J_{r_{n}}^{A_{l}} x_{n}-p\right\| \\
& \leqslant \sum_{i=1}^{l} a_{i}\left\|J_{r_{n}}^{A_{l}} x_{n}-p\right\| \leqslant \sum_{i=1}^{l} a_{i}\left\|x_{n}-p\right\|=\left\|x_{n}-p\right\|,
\end{aligned}
$$

and

$$
\begin{aligned}
\left\|x_{n+1}-p\right\| & =\left\|\alpha_{n} f\left(x_{n}\right)+\left(1-\alpha_{n}\right) y_{n}-p\right\| \\
& \leqslant \alpha_{n}\left\|f\left(x_{n}\right)-p\right\|+\left(1-\alpha_{n}\right)\left\|y_{n}-p\right\| \\
& \leqslant \alpha_{n}\left\|f\left(x_{n}\right)-f(p)\right\|+\alpha_{n}\|f(p)-p\|+\left(1-\alpha_{n}\right)\left\|x_{n}-p\right\| \\
& \leqslant \alpha_{n} \alpha\left\|x_{n}-p\right\|+\alpha_{n}\|f(p)-p\|+\left(1-\alpha_{n}\right)\left\|x_{n}-p\right\| \\
& =\left(1-\alpha_{n}(1-\alpha)\left\|x_{n}-p\right\|+\alpha_{n}(1-\alpha) \frac{\|f(p)-p\|}{1-\alpha}\right. \\
& \leqslant \max \left\{\left\|x_{n}-p\right\|, \frac{\|f(p)-p\|_{1}}{1-\alpha} .\right.
\end{aligned}
$$

By induction, for all $n \geqslant 0$, we obtain

$$
\left\|x_{n}-p\right\| \leqslant \max \left\{\left\|x_{0}-p\right\|, \frac{\|f(p)-p\|}{1-\alpha}\right\} .
$$

Therefore, the sequence $\left\{x_{n}\right\}$ is bounded, and so are $\left\{y_{n}\right\},\left\{f\left(x_{n}\right)\right\}$. 
Step 2. We show that $\left\|x_{n+1}-x_{n}\right\| \rightarrow 0, n \rightarrow \infty$. We calculate $\left\|y_{n+1}-y_{n}\right\|$ firstly.

From (1.1), we know

$$
\begin{aligned}
\left\|y_{n}-y_{n-1}\right\| & =\left\|a_{1}\left(J_{r_{n}}^{A_{1}} x_{n}-J_{r_{n-1}}^{A_{1}} x_{n-1}\right)+a_{2}\left(J_{r_{n}}^{A_{2}} x_{n}-J_{r_{n-1}}^{A_{2}} x_{n-1}\right)+\cdots+a_{l}\left(J_{r_{n}}^{A_{l}} x_{n}-J_{r_{n-1}}^{A_{l}} x_{n-1}\right)\right\| \\
& \leqslant \sum_{i=1}^{l} a_{i}\left\|J_{r_{n}}^{A_{i}} x_{n}-J_{r_{n-1}}^{A_{i}} x_{n-1}\right\| .
\end{aligned}
$$

Lemma 2.3 (The resolvent identity) implies that

$$
J_{r_{n}}^{A_{i}} x_{n}=J_{r_{n-1}}^{A_{i}}\left(\frac{r_{n-1}}{r_{n}} x_{n}+\left(1-\frac{r_{n-1}}{r_{n}}\right) J_{r_{n}}^{A_{i}} x_{n}\right) .
$$

If $r_{n-1} \leqslant r_{n}$, using the resolvent identity

$$
\begin{aligned}
\left\|J_{r_{n}}^{A_{i}} x_{n}-J_{r_{n-1}}^{A_{i}} x_{n-1}\right\| & =\left\|J_{r_{n-1}}^{A_{i}}\left(\frac{r_{n-1}}{r_{n}} x_{n}+\left(1-\frac{r_{n-1}}{r_{n}}\right) J_{r_{n}}^{A_{i}} x_{n}\right)-J_{r_{n-1}}^{A_{i}} x_{n-1}\right\| \\
& \leqslant\left\|\frac{r_{n-1}}{r_{n}} x_{n}+\left(1-\frac{r_{n-1}}{r_{n}}\right) J_{r_{n}}^{A_{i}} x_{n}-x_{n-1}\right\| \\
& \leqslant\left\|x_{n}-x_{n-1}\right\|+\left|1-\frac{r_{n-1}}{r_{n}}\right|\left\|J_{r_{n}}^{A_{i}} x_{n}-x_{n-1}\right\| \\
& \leqslant\left\|x_{n}-x_{n-1}\right\|+\frac{\left|r_{n}-r_{n-1}\right|}{\varepsilon}\left\|J_{r_{n}}^{A_{i}} x_{n}-x_{n-1}\right\| .
\end{aligned}
$$

Substituting (3.2) into (3.1), we obtain

$$
\begin{aligned}
\left\|y_{n}-y_{n-1}\right\| & =\sum_{i=1}^{l} a_{i}\left(\left\|x_{n}-x_{n-1}\right\|+\frac{\left|r_{n}-r_{n-1}\right|}{\varepsilon}\left\|J_{r_{n}}^{A_{i}} x_{n}-x_{n-1}\right\|\right) \\
& \leqslant\left\|x_{n}-x_{n-1}\right\|+\left|r_{n}-r_{n-1}\right| M_{1},
\end{aligned}
$$

where $M_{1}$ is a constant such that

$$
M_{1}=\sup \left\{\frac{\left\|J_{r_{n}}^{A_{i}} x_{n}-x_{n-1}\right\|}{\varepsilon}, i=1,2, \cdots, l\right\} .
$$

On the other hand, we have

$$
x_{n+1}-x_{n}=\alpha_{n} f\left(x_{n}\right)+\left(1-\alpha_{n}\right) y_{n}-\alpha_{n-1} f\left(x_{n-1}\right)+\left(1-\alpha_{n-1}\right) y_{n-1} .
$$

So, from (3.3) and (3.4), we have

$$
\begin{aligned}
\left\|x_{n+1}-x_{n}\right\|= & \| \alpha_{n}\left(f\left(x_{n}\right)-f\left(x_{n-1}\right)\right)+\left(1-\alpha_{n}\right)\left(y_{n}-y_{n-1}\right) \\
& +\left(\alpha_{n}-\alpha_{n-1}\right)\left(f\left(x_{n-1}\right)-y_{n-1}\right) \| \\
\leqslant & \alpha_{n}\left\|f\left(x_{n}\right)-f\left(x_{n-1}\right)\right\|+\left(1-\alpha_{n}\right)\left\|y_{n}-y_{n-1}\right\|+\left|\alpha_{n}-\alpha_{n-1}\right|\left\|f\left(x_{n-1}\right)-y_{n-1}\right\| \\
\leqslant & \alpha_{n} \alpha\left\|x_{n}-x_{n-1}\right\|+\left(1-\alpha_{n}\right)\left(\left\|x_{n}-x_{n-1}\right\|\right. \\
& \left.+\left|r_{n}-r_{n-1}\right| M_{1}\right)+\left|\alpha_{n}-\alpha_{n-1}\right|\left\|f\left(x_{n-1}\right)-y_{n-1}\right\| \\
\leqslant & \left(1-\alpha_{n}(1-\alpha)\right)\left\|x_{n}-x_{n-1}\right\|+M_{2}\left(\left|r_{n}-r_{n-1}\right|+\left|\alpha_{n}-\alpha_{n-1}\right|\right),
\end{aligned}
$$

where $M_{2}$ is a constant such that

$$
M_{2}=\sup \left\{M_{1},\left\|f\left(x_{n-1}\right)-y_{n-1}\right\|\right\} .
$$

By assumptions (i), (ii), we have that

$$
\lim _{n \rightarrow \infty} \alpha_{n}=0, \quad \sum_{n=0}^{\infty} \alpha_{n}(1-\alpha)=\infty, \quad \sum_{n=1}^{\infty}\left(\left|r_{n}-r_{n-1}\right|+\left|\alpha_{n}-\alpha_{n-1}\right|\right)<\infty
$$

Hence, Lemma 2.2 is applicable to (3.5) and we obtain

$$
\left\|x_{n+1}-x_{n}\right\| \rightarrow 0, \quad n \rightarrow \infty .
$$


Step 3. We show that $\limsup _{n \rightarrow \infty}\left\langle(I-f) Q(f), J_{\varphi}\left(Q(f)-x_{n}\right)\right\rangle \leqslant 0$, where $Q(f)=\lim _{t \rightarrow 0} z_{t}$. By using $\alpha_{n} \rightarrow 0(n \rightarrow \infty),\left\{y_{n}\right\},\left\{f\left(x_{n}\right)\right\}$ are bounded, we have

$$
\left\|x_{n+1}-y_{n}\right\|=\alpha_{n}\left\|f\left(x_{n}\right)-y_{n}\right\| \rightarrow 0, \quad n \rightarrow \infty .
$$

By virtue of $\left\|x_{n+1}-x_{n}\right\| \rightarrow 0,\left\|x_{n+1}-y_{n}\right\| \rightarrow 0(n \rightarrow \infty)$, we have

$$
\left\|x_{n}-y_{n}\right\| \leqslant\left\|x_{n}-x_{n+1}\right\|+\left\|x_{n+1}-y_{n}\right\| \rightarrow 0 .
$$

Let $S_{r_{n}}=a_{1} J_{r_{n}}^{A_{1}}+a_{2} J_{r_{n}} A_{2}+\cdots+a_{l} J_{r_{n}} A_{l}$. From (1.1), we know

$$
y_{n}=S_{r_{n}} x_{n} .
$$

Combining (3.6) and (3.7), we obtain

$$
\left\|x_{n}-S_{r_{n}} x_{n}\right\| \rightarrow 0, \quad n \rightarrow \infty
$$

From Lemma 2.6, we know $S_{r_{n}}$ is nonexpansive and $F\left(S_{r_{n}}\right)=\bigcap_{i=1}^{l} N\left(A_{i}\right)$. Since $E$ is reflexive Banach space and $\left\{x_{n}\right\}$ is bounded, we may further assume that there exists a subsequence $\left\{x_{n_{k}}\right\}$

$$
x_{n_{k}} \rightarrow \bar{x}
$$

By using of (3.8), (3.9) and Lemma 2.4, we obtain

$$
\bar{x} \in F\left(S_{r_{n}}\right)=\bigcap_{i=1}^{l} N\left(A_{i}\right) .
$$

From Lemma 2.5, we know $z_{\mathrm{t}}=\operatorname{tf}\left(z_{\mathrm{t}}\right)+(1-\mathrm{t}) S_{\mathrm{r}_{\mathrm{n}}} z_{\mathrm{t}}$ convergence strongly to a point in $\mathrm{Q}(\mathrm{f}) \in \mathrm{F}\left(\mathrm{S}_{\mathrm{r}_{\mathrm{n}}}\right)=$ $\bigcap_{i=1}^{l} N\left(A_{i}\right)$, as $t \rightarrow 0$ and

$$
\left\langle(I-f) Q(f), J_{\varphi}(Q(f)-p)\right\rangle \leqslant 0, \quad p \in F\left(S_{r_{n}}\right) .
$$

So from (3.9), (3.10), (3.11), we know that

$$
\begin{aligned}
\limsup _{n \rightarrow \infty}\left\langle(I-f) Q(f), J_{\varphi}\left(Q(f)-x_{n}\right)\right\rangle & =\lim _{k \rightarrow \infty}\left\langle(I-f) Q(f), J_{\varphi}\left(Q(f)-x_{n_{k}}\right)\right\rangle \\
& =\left\langle(I-f) Q(f), J_{\varphi}(Q(f)-\bar{x})\right\rangle \leqslant 0 .
\end{aligned}
$$

Step 4. Finally we show that $x_{n} \rightarrow Q(f)$. We apply Lemma 2.1 to get

$$
\begin{aligned}
\phi\left(\left\|x_{n+1}-Q(f)\right\|\right)= & \phi\left(\left\|\alpha_{n} f\left(x_{n}\right)+\left(1-\alpha_{n}\right) S_{r_{n}} x_{n}-Q(f)\right\|\right) \\
= & \phi\left(\left\|\alpha_{n}\left(f\left(x_{n}\right)-Q(f)\right)+\left(1-\alpha_{n}\right)\left(S_{r_{n}} x_{n}-Q(f)\right)\right\|\right) \\
= & \phi\left(\left\|\alpha_{n}\left(f\left(x_{n}\right)-f(Q(f))\right)+\alpha_{n}(f(Q(f))-Q(f))+\left(1-\alpha_{n}\right)\left(S_{r_{n}} x_{n}-Q(f)\right)\right\|\right) \\
\leqslant & \phi\left(\left\|\left(1-\alpha_{n}\right)\left(S_{r_{n}} x_{n}-Q(f)\right)+\alpha_{n}\left(f\left(x_{n}\right)-f(Q(f))\right)\right\|\right) \\
& +\alpha_{n}\left\langle f(Q(f))-Q(f), J_{\varphi}\left(x_{n+1}-Q(f)\right)\right\rangle \\
\leqslant & \phi\left(\left(1-\alpha_{n}\right)\left\|x_{n}-Q(f)\right\|+\alpha_{n} \alpha\left\|x_{n}-Q(f)\right\|\right)+\alpha_{n}\left\langle f(Q(f))-Q(f), J_{\varphi}\left(x_{n+1}-Q(f)\right)\right\rangle \\
\leqslant & \left(1-\alpha_{n}(1-\alpha)\right) \phi\left(\left\|x_{n}-Q(f)\right\|\right)+\alpha_{n}\left\langle(I-f) Q(f), J_{\varphi}\left(Q(f)-x_{n+1}\right)\right\rangle .
\end{aligned}
$$

By using of (3.12), $\lim _{n \rightarrow \infty} \alpha_{n}=0, \sum_{n=0}^{\infty} \alpha_{n}(1-\alpha)=\infty$, Lemma 2.2, we have

$$
\phi\left(\left\|x_{n}-Q(f)\right\|\right) \rightarrow 0, \quad n \rightarrow \infty \text {. }
$$

That is

$$
\begin{gathered}
\left\|x_{n}-Q(f)\right\| \rightarrow 0, \quad n \rightarrow \infty \\
x_{n} \rightarrow Q(f), \quad n \rightarrow \infty .
\end{gathered}
$$

The proof is completed. 
Corollary 3.2. Let $A_{i}: K \rightarrow E, i=1,2, \cdots$, l, be a finite family of m-accretive operators such that $\bigcap_{i=1}^{l} N\left(A_{i}\right) \neq$ $\emptyset$ and the rest conditions be the same as in Theorem 3.1. Then the conclusion of Theorem 3.1 still holds.

Corollary 3.3. Assume that $\mathrm{E}$ is strictly convex reflexive Banach space with a weakly continuous duality mapping $\mathrm{J}_{\varphi}$ associated to a gauge $\varphi$. Let $\mathrm{K} \subset \mathrm{E}$ be a nonempty closed convex subset and $\mathrm{f}: \mathrm{K} \rightarrow \mathrm{K}$ be a contractive mapping with the contractive coefficient $\alpha \in(0,1),\left\{T_{i}: K \rightarrow E, i=1,2, \cdots, l\right\}$ be a finite family of nonexpansive operators such that $\bigcap_{i=1}^{l} \mathrm{~F}\left(\mathrm{~T}_{i}\right) \neq \emptyset$. For any $\mathrm{x}_{0} \in \mathrm{K}$, suppose that $\left\{\mathrm{x}_{n}\right\}$ is defined by the modified viscosity iteration

$$
\left\{\begin{aligned}
y_{n} & =\beta_{1} T_{1} x_{n}+\beta_{2} T_{2} x_{n}+\cdots+\beta_{l} T_{l} x_{n}, \\
x_{n+1} & =\alpha_{n} f\left(x_{n}\right)+\left(1-\alpha_{n}\right) y_{n}, \quad n \geqslant 0,
\end{aligned}\right.
$$

where $0<\beta_{i}<1$, for $i=1,2, \cdots, l, \sum_{i=1}^{l} \beta_{i}=1$ and $\left\{\alpha_{n}\right\} \subset(0,1)$. We assume that the following mild conditions on the sequences of parameters are established:

- $\lim _{n \rightarrow \infty} \alpha_{n}=0, \quad \sum_{n=0}^{\infty} \alpha_{n}=\infty, \quad \sum_{n=0}^{\infty}\left|\alpha_{n+1}-\alpha_{n}\right|<\infty$.

Then $\left\{x_{n}\right\}$ converges strongly to a common fixed point of $T_{i}, i=1,2, \cdots, l$.

Proof. We only need to replace $\mathrm{J}_{r_{n}}^{A_{i}}$ with $T_{i}$ in the proof of Theorem 3.1.

Remark 3.4. In Theorem 3.1, if $\mathrm{f} \equiv u, \mathrm{a}_{2}=\cdots=\mathrm{a}_{\mathrm{l}}=0, \mathrm{a}_{1}=1$, then the sequence $\left\{x_{n}\right\}$ defined by (1.1) induces $x_{n+1}=\alpha_{n} u+\left(1-\alpha_{n}\right) J_{r_{n}}^{A_{1}} x_{n}$ and the results of Theorem 3.1 are the main results in References [18] and [6]. If $A_{1}=0, f \equiv u, a_{3}=\cdots=a_{l}=0, a_{1}=a_{n}, a_{2}=1-a_{n}$, then the sequence $\left\{x_{n}\right\}$ defined by (1.1) turns to

$$
\left\{\begin{aligned}
y_{n} & =a_{n} x_{n}+\left(1-a_{n}\right) J_{r_{n}}^{A_{2}} x_{n}, \\
x_{n+1} & =\alpha_{n} u+\left(1-\alpha_{n}\right) y_{n},
\end{aligned}\right.
$$

and the results of Theorem 3.1 are the main results in [10]. If $\equiv u, A_{1}=0$, then the sequence $\left\{x_{n}\right\}$ defined by (1.1) changes as

$$
\left\{\begin{array}{c}
x_{n+1}=\alpha_{n} u+\left(1-\alpha_{n}\right) S_{r_{n}} x_{n}, \\
S_{r_{n}}=a_{1} I+a_{2} J_{r_{n}}^{A_{2}}+\cdots+a_{l} J_{r_{n}}^{A_{l}},
\end{array}\right.
$$

and the results of Theorem 3.1 are the main results in [14].

So, in some ways, the results here improve and extend many corresponding recent results in ([1$4,6,7,10,11,14,18])$.

\section{Acknowledgment}

The authors are extremely grateful to the editors and referees for useful comments and suggestions that improved the content of the article. This work was supported by the National Natural Science Foundation of China (Grant no. 11671365) and the Natural Science Foundation of Zhejiang Province (Grant no. LY14A010011).

\section{References}

[1] L. C. Ceng, A. R. Khan, Q. H. Ansari, J. C. Yao, Strong convergence of composite iterative schemes for zeros of m-accretive operators in Banach spaces, Nonlinear Anal., 70 (2009), 1830-1840. 2.3, 3.4

[2] R. D. Chen, Y. J. Liu, X. L. Shen, Iterative approximation of a zero of accretive operator in Banach space, Nonlinear Anal., 71 (2009), 346-350. 1

[3] R. D. Chen, Z. C. Zhu, Viscosity approximation fixed point for nonexpansive and m-accretive operators, Fixed Point Theory Appl., 2006 (2006), 10 pages. 1

[4] R. D. Chen, Z. C. Zhu, Viscosity approximation fixed point for accretive operator in Banach space, Nonlinear Anal., 69 (2008), 1356-1363. 1, 3.4 
[5] Y. J. Cho, X. L. Qin, Viscosity approximation methods for a family of m-accretive mappings in reflexive Banach spaces, Positivity, 12 (2008), 483-494. 2.5

[6] L. G. Hu, L. W. Liu, A new iterative algorithm for common solutions of a finite family of accretive operators, Nonlinear Anal., 70 (2009), 2234-2351. 3.4

[7] J. S. Jung, Convergence of composite iterative methods for finding zeros of accretive operators, Nonlinear Anal., 71 (2009), 1736-1746. 1, 3.4

[8] T. H. Kim, H. K. Xu, Strong convergence of modified Mann iterations, Nonlinear Anal., 61 (2005), 51-60. 1

[9] T. C. Lim, H. K. Xu, Fixed point theorems for asymptotically nonexpansive mappings, Nonlinear Anal., 22 (1994), 1345-1355. 2

[10] A. Moudafi, Viscosity approximation methods for fixed-points problems, J. Math. Anal. Appl., 241 (2000), 46-55. 1, 3.4

[11] Y. H. Wang, S. Chebbi, H. K. Xu, Weak convergence of an iterative format for finding common zeros of two accretive operators in Banach spaces, J. Nonlinear Convex Anal., 16 (2015), 1937-1947. 1, 3.4

[12] Y. H. Wang, Y. H. Xia, Strong convergence for asymptotically pseudocontractions with the demiclosedness principle in Banach spaces, Fixed Point Theory Appl., 2012 (2012), 8 pages. 2.4

[13] H. K. Xu, Iterative algorithms for nonlinear operator, J. London Math. Soc., 66 (2002), 240-256. 2.2

[14] H. K. Xu, Viscosity approximation methods for nonexpansive mappings, J. Math. Anal. Appl., 298 (2004), 279-291. 1, 3.4

[15] H. K. Xu, Strong convergence of an iterative method for nonexpansive and accretive operators, J. Math. Anal. Appl., 314 (2006), 631-643. 1

[16] Y. H. Yao, N. Shahzad, Strong convergence of a proximal point algorithm with general errors, Optim. Lett., 6 (2012), 621-628. 1

[17] Y. H. Yao, N. Shahzad, Y. C. Liou, Modified semi-implicit midpoint rule for nonexpansive mappings, Fixed Point Theory Appl., 2015 (2015), 15 pages. 1

[18] H. Zegeye, N.Shahzad, Strong convergence theorems for a common zero of a finite family of m-accretive mapping, Nonlinear Anal., 66 (2007), 1161-1169. 1, 3.4

[19] Q. Yuan, S. Lv, Strong convergence of a parallel iterative algorithm in a reflexive banach space, Fixed Point Theory Appl., 2014 (2014), 1-9. 2.1 